\title{
Electrical Property of Carbon Nanotube Fibers from Chemical Vapor Deposition Synthesis
}

\author{
Zhu Jingdong* \\ Key Laboratory of Advanced Ceramics and Machining Technology, Ministry of Education, School of \\ Material Science and Engineering, Tianjin University, Tianjin 300072, People's Republic of China
}

*Corresponding author Email: zhujing_dong@126.com

Keywords: carbon nanotube, carbon nanotube fiber, electrical property

\begin{abstract}
Because of facile synthesis, carbon nanotube (CNT) fibers from chemical vapor deposition can be used for diverse applications. In this study, the electrical property of CNT fibers was investigated. The room-temperature electrical conductivity of a typical CNT fiber was measured to be $5 \times 10^{5} \mathrm{~S} / \mathrm{m}$. The electrical-conductivity transport mechanism of CNT fibers was elucidated according to the temperature dependence of the resistance, indicating that the Mott's variable-range hopping mechanism operates Moreover, the thermoelectric power was measured, indicating that the carrier within the fiber is a hole.
\end{abstract}

\section{Introduction}

Carbon nanotubes (CNTs) with unique one-dimensional nanostructures possess super strength[1], high electrical[2] and thermal conductivities [3], and multifunctional properties. CNT fiber, an oriented assembly of CNTs, has several potential applications such as artificial muscle[4], supercapacitor[5], ultrahigh conductivity fiber[6], stretchable electrical conductor[7], and high-performance structural fibers[8].Currently, there are four main routes to synthesize CNT fibers: 1) by spinning from a CNT solution[9], 2) by spinning from an aligned CNT array[10], 3) by spinning from a CNT aerogel synthesized by chemical vapor deposition (CVD) $[11,12]$, 4) by twisting/rolling from a CNT film[13]. CNT fibers synthesized by CVD can be commercially available on a large scale, because of facile synthesis and a stable continuously spinnable process. Currently, the fabrication technology of CVD CNT fibers has matured; however, the properties of CNT fibers should be evaluated for their applications, particularly the electrical property of CNT fibers. In this study, the electrical property of CNT fibers was investigated.

\section{Experimental}

CNT fibers were synthesized by the CVD method. A mixture of carbon source and catalyst was injected into a high-temperature reactor along with $\mathrm{H}_{2}$ stream, affording a continuous stream of CNT fiber at the bottom of the reactor. The CNT fiber was removed from the reactor after online fiber densification with acetone A more detailed description of the synthesis process can be found elsewhere[12]. The cross-section and surface morphologies of the CNT fibers were characterized using a scanning electron microscope (JSM-6700F), tunneling electron microscope (Tecnai-G20 F20), and Raman spectrometer (Renishaw).

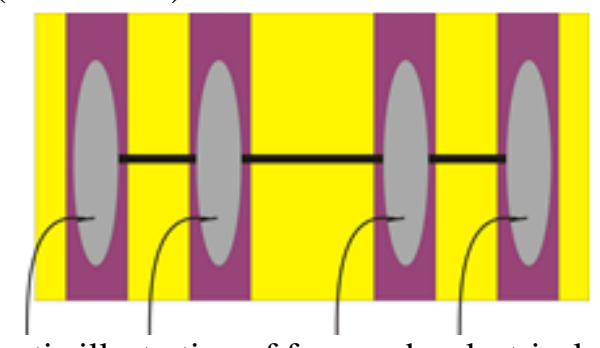

Fig. 1.Schematic illustration of four-probe electrical measurement

Electrical measurement was conducted using the four-probe method at temperatures from 2 to $300 \mathrm{~K}$. The constant direct current passing through the fiber was set at $20 \mu \mathrm{A}$. To establish a good 
contact between the fiber and electrode, a prepatterned glass substrate with four Al electrode strips was prepared by sputtering through a shadow mask. The four Al strips were $1 \mathrm{~mm}$ wide, $5 \mathrm{~mm}$ long, and separated by $1 \mathrm{~mm}$. Then, the fiber was transferred onto the prepatterned substrate as shown in Fig. 1 and covered with a thin layer of silver paste at each Al electrode. For the thermoelectric power (TEP) measurement, the two ends of a strand (length $3 \mathrm{~cm}$ ) were attached to hot and cold copper plates using the silver paste. Then, the hot plate was heated, and a constant temperature gradient $\left(\Delta \mathrm{T}=\mathrm{T}_{\text {high }}-\mathrm{T}_{\text {low }}=5 \mathrm{~K}\right)$ was established along the direction of the length of the fiber. The voltage difference $(\Delta \mathrm{V})$ between the two ends of the fiber was measured simultaneously using a Keithley 2400 nanovoltmeter with two copper wires welded to the hot and cold copper plates. Therefore, the TEP can be obtained as follows: $\mathrm{S}=\Delta \mathrm{V} / \Delta \mathrm{T}$.

\section{Results and discussion}

\section{Structure of CNT fiber}

The CNT fibers were continuous and soft such as cotton yarns. Three hierarchy structures were observed in the scanning electron microscope (SEM) and high-resolution transmission electron microscope (HRTEM). At the macroscopic scale, the fiber is hollow, with a fiber wall of 1-10 $\mu \mathrm{m}$ (Fig. 2a). The fiber wall consists of large quantity of CNT bundles with a diameter of 10-100 nm, slightly aligned with the fiber axis (Fig. 2b). At the microscopic scale, the bundles comprise of large-diameter double-wall CNT stacks (Fig. 2c). The crystallization of the fiber was characterized by Raman spectroscopy, $\mathrm{I}_{\mathrm{G}} / \mathrm{I}_{\mathrm{D}}$ value of 6.3 was obtained (Fig. $2 \mathrm{~d}$ ), indicating that the CNT fibers have less defect than those obtained from array[14].
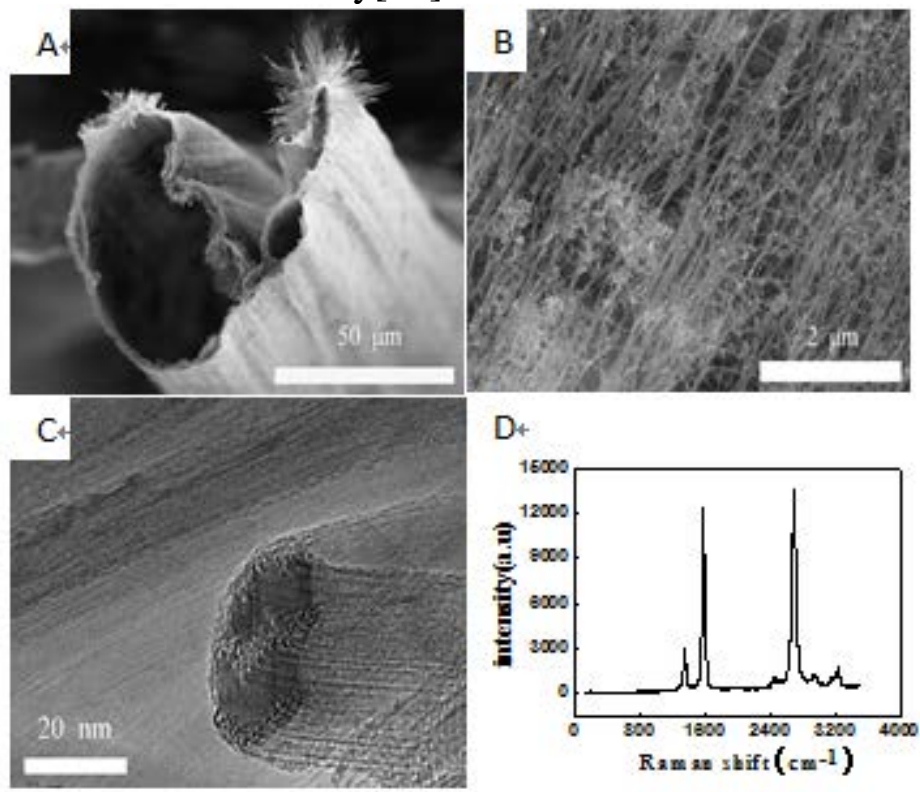

Fig. 2. a) Low-magnification scanning electron microscopy (SEM) image of a typical CNT fiber. b) High-magnification SEM image. c) Cross-sectional high-resolution transmission electron microscopy image. d) Raman spectrum of the fiber.

\section{Electrical property of CNT fiber}

To determine the electrical conductivity of the CNT fiber using the formula $\rho=(\mathrm{RS}) / \mathrm{L}$, their cross-section area should be determined first. However, it is difficult to measure the cross-section area directly because of their irregularity. Therefore, the cross-section area was obtained by an indirect method-by measuring the linear density through the vibration principle-as typically employed in the spinning industry. The cross-section area was calculated by dividing the linear density with the volume density of the graphite $\left(2.1 \mathrm{~g} / \mathrm{cm}^{3}\right)$. 


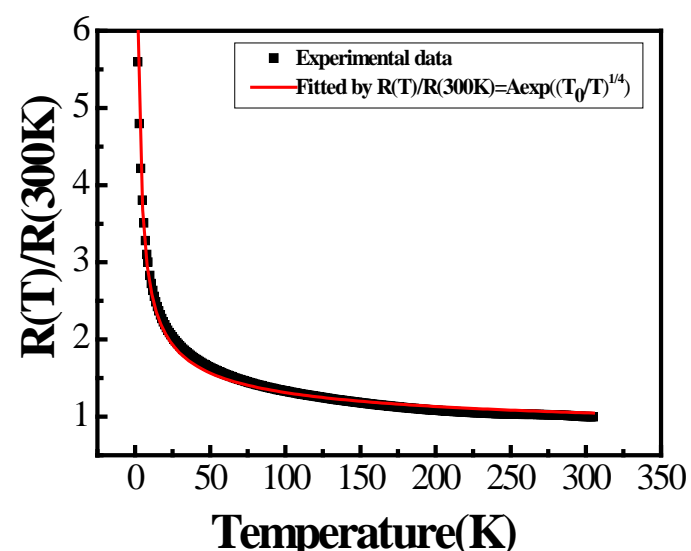

Fig. 3. Temperature dependence of fiber resistance R(T), normalized with respect to the R (300 K) and the fitting of resistance data with the VRH mechanism

The electrical resistivity of a typical CNT fiber is $2 \times 10^{-6} \Omega \mathrm{m}$ at room temperature, and the corresponding conductivity is $5 \times 10^{5} \mathrm{~S} / \mathrm{m}$, which is higher than the CNT fiber from array[14], but lower than the CNT fiber from wetting spinning[6]. To understand the electrical-conductivity transport mechanism within CNT fiber, the temperature dependence of the electrical resistivity of the CNT fiber was measured. Fig. 3 shows the temperature dependence of the resistance from 2 to $300 \mathrm{~K}$. The resistance decreased monotonically and smoothly with the increase in temperature, indicating the semiconducting behavior. The temperature dependence of resistance can help to understand the conduction mechanism. The plot of resistance versus temperature was found to be consistent with the Mott's three-dimensional variable-range hopping mechanism, which can be expressed as follows: $R(T) / R(300 \mathrm{~K})=A_{0} \exp \left[\left(T_{0} / T\right)^{1 / 4}\right]$, where $A_{0}$ and $T_{0}$ are constants. This is probably because of the contact between CNT bundles[14]. Furthermore, to determine the type of electrical carrier within the fiber, the TEP of the fiber was measured to be $+65 \mu \mathrm{V} / \mathrm{K}$. The positive sign of the TEP value indicates that the electrical carrier is a hole, similar to the P-type semiconductor.

\section{Conclusion}

The CNT fibers from CVD process were found to compose of CNT bundles with an electrical conductivity of $5 \times 10^{5} \mathrm{~S} / \mathrm{m}$ at room temperature. The temperature dependence of the resistance of the CNT fiber indicates that the 3D variable-range hopping mechanism operates. The TEP measurement reveals that the electrical carriers within the fiber are holes.

\section{Acknowledge}

Author is grateful to professor Ya-Li Li at Tianjin University for providing CNT fibers from CVD and Professor Li Zhiqing at Tianjin University for his help with electrical measurements

\section{Reference}

[1] Yu MF, Files BS, Arepalli S, Ruoff RS. Tensile loading of ropes of single wall carbon nanotubes and their mechanical properties. Phys Rev Lett 2000; 84(24): 5552-55.

[2] Collins PG and Avouris P, Nanotubes for electronics. Scientific American 2000; 283(6): 62.

[3] Balandin AA, Thermal properties of graphene and nanostructured carbon materials. Nat Mater 2011; 10(8): 569-81.

[4] Lima MD, li N, Endrade MJ, et al. Electrically, chemically, and photonically powered torsional and tensile actuation of hybrid carbon nanotube yarn muscles. Science 2012; 338: 928-32. 
[5] Dalton AB, Collins S, Munoz E, et al. Super-tough carbon-nanotube fibres. Nature 2003; 423(6941): 703.

[6] Behabtu N, Young CC, Tsentalovich DE, et al. Strong, light, multifunctional fibers of carbon nanotubes with ultrahigh conductivity. Science 2013; 339: 182-6.

[7] Zu M, Li QW, Wang GJ, et al. Carbon nanotube fiber based stretchable conductor. Adv Func Mater 2013; 23: 789-93.

[8] Koziol K, Vilatela J, Moisala A, et al. High-Performance carbon nanotube fiber. Science 2007; 318: 1892-95.

[9] Ericson LM, Fan H, Peng HQ, et al. Macroscopic, neat, single-walled carbon nanotube fibers. Science 2004; 305(5689): 1447-50.

[10] Zhang M, Atkinson KR, Baughman RH, Multifunctional carbon nanotube yarns by downsizing an ancient technology. Science 2004; 306(5700): 1358-61.

[11] Li YL, Kinloch IA, and Windle AH, Direct spinning of carbon nanotube fibers from chemical vapor deposition synthesis. Science 2004; 304(5668): 276-8.

[12] Zhong XH, Li YL, Liu YK, et al. Continuous multilayered carbon nanotube yarns. Adv Mater 2010; 22(6): 692-6.

[13] Ma WJ, Liu LQ, Yang R, et al. Monitoring a micromechanical process in macroscale carbon nanotube films and fibers. Adv Mater 2009; 21(5): 603-8.

[14] Li QW, Li Y, Zhang XF, Chikkannaavar SB et al. Structure-dependent electrical properties of carbon nanotube fibers. Adv Mater 2007; 19(20): 3358-63. 